\title{
INTENSITY CALIBRATION IN THE PRESENCE OF A DISTURBING EFFECT DEPENDENT ON WAVELENGTH
}

\author{
P. KOTRČ \\ Astronomical Institute \\ 25165 Ondrejov \\ Czech Republic
}

Conversion of light detector signals to intensity values is one of the most important factors influencing precision of spectroscopic observations. Most of the classical light detectors used in astronomical practice are more or less nonlinear. As the photoemulsion has long been the most widespread nonlinear light detector, many improvements in the calibration methods concerned its nonlinearity. In addition to it, there are other substantial sources of inaccuracy in the calibration process of real astronomical images and spectrograms. They are mostly related to real light conditions in telescopes and spectrographs, as well as to the wavelength dependent sensitivity of light detectors. Some of these factors can be taken into account and involved in the calibration process. Similar effects are considered when a flat-field is evaluated for CCD detectors or when image structure varies over a photographic plate.

Apparent complications arise due to a variable level of parasitic light occurring in the spectrum, especially in some types of spectrographs, principally when it is not possible to exclude these effects during observations. Then, we have to find regular calibration of such images with a systematical occurrence of a parasitic light or a detector disturbance. Beckers et al. (1972) warned about possible errors in the calibration process due to the influence of scattered light inside the spectrograph. Actually, this is the situation of our Echelle-type solar spectra. Due to setting the monochromator or other reasons, the scattered or parasitic light is present; and it behaves as a disturbing factor, slightly varying along the wavelength direction. The conversion function (or calibration curve) is determined and therefore valid only for a fixed wavelength. When a broader wavelength region is studied, the wavelength dependent sensitivity of light detectors has to be taken into account. Then a number of individual characteristic curves is evaluated for individual wavelength regions. Generally, both these effects show some similarity and up to a certain level can be considered as disturbing factors.

For high dispersion solar spectra that we have observed at the Echelle spectrograph of the Sacramento Peak Vacuum Tower Telescope, $70 \mathrm{~mm}$ spectral strips are typical. Thus only narrow spectral regions with individual lines and their close vicinity can be detected. In the H $\alpha$ region,

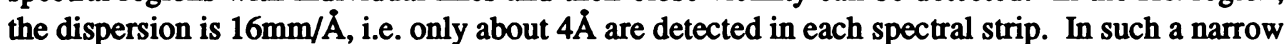
region it is practically impossible to find the spectral continuum and to use it for absolute calibration. Instead of the continuum, we decided to use the quiet solar disk centre profiles of Balmer series lines as measured by David (1961). To calibrate the spectral data, we related the detected signal of the quiet solar disk centre passing through attenuating wedges to David's 
profiles. All blended spectral segments were excluded in the calibration.

The usual polynomial calibration formula for the relative intensity $I_{R}$ is:

$$
\log I_{R}(\lambda)=\sum_{i=0}^{3} a_{i} D^{i}
$$

where $a_{i}$ are the coefficients of a relative calibration curve and $D$ is the measured photographic density.

Though this calibration curve is a good approximation of the conversion function, there appear some differences. As can be seen in Fig. 1, there is a systematic shift of the approximated calibrated profiles in relation to the profiles of David and those from the Solar atlas of the University at Liege. The differences in intensity (bottom) increase slightly along the wavelength axis. The intensity difference along the profile is up to $\pm 5 \%$. This effect is the disturbing factor caused by the presence of parasitic or scattered light. Assuming that the disturbing effect is linearly dependent on the wavelength $\lambda$ (or generally on the position of the image), we obtain for the absolute intensity $I_{A}(\lambda)$ :

$$
\log \left[I_{A}(\lambda) T(\lambda)\right]=b_{0}+b_{1} \lambda+\sum_{i=1}^{3} a_{i} D^{l}
$$

where $T(\lambda)$ is the resulting transparency of an individual step wedge combined with the transparency of a neutral attenuating filter.

Thus, the present linear disturbing factor is involved in the expression $a_{0}=b_{0}+b_{1} \lambda$. As a consequence, instead of 4 parameters of the characteristic curve (Equ. 1), we evaluate 5 coefficients (Equ. 2). All the coefficients are evaluated in two approximations by the method of least squares.

The resulting calibration can be seen in Fig. 2. We can see that the systematic increase of the intensity has practically disappeared and the residual difference is of the order $\pm 1 \%$. We are convinced that the proposed procedure can be used in other examples of real astronomical images and spectrograms where similar linearly dependent disturbing effects occur.

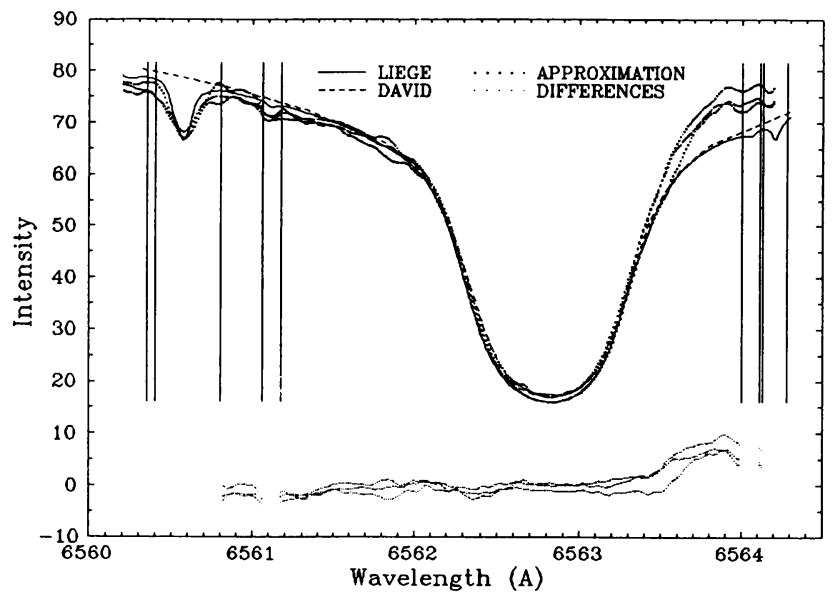

Figure 1. First approximation of the calibrated profiles (dotted) shows systematic differences (dotted below) to the standard profiles of Liege (solid) and David's (dashed). Vertical lines mark blends on the profile. 


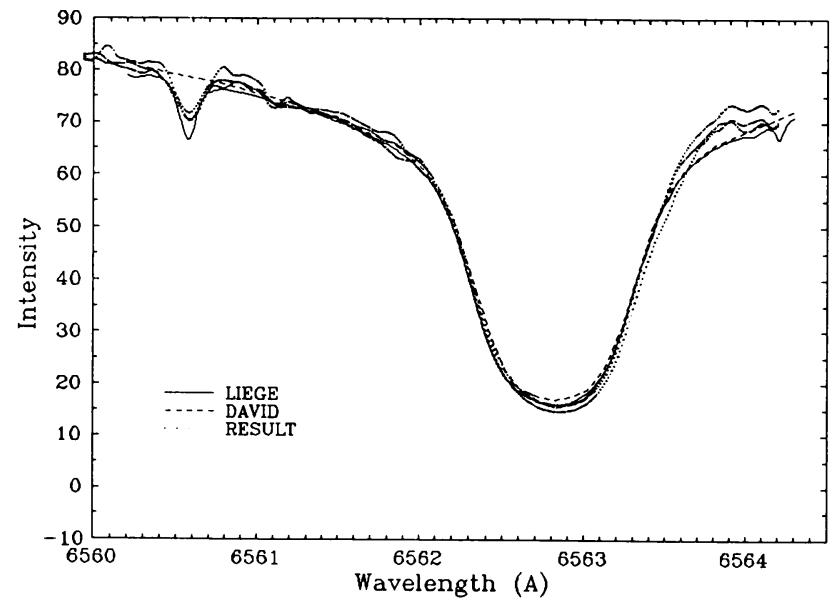

Figure 2. Profiles (dotted) calibrated by the improved calibrating curve are better fitted to the profiles of Atlas Liege (solid) and David's (dashed).

\section{References}

Beckers, J.M., Mauter, H.A. and Mann, R.G., 1972. Solar Phys., 25, 81.

David, K.H., 1961. Z. Astrophys., 53, 37. 